one-third of the RA patients had osteoporosis (OP). However, most fragility fractures occur in patients not suffering from OP, that can be partly explained by impaired quality of bone, which is not measured with DXA. Therefore, only the measurement of bone mineral density is not sufficient to determine the indication for OP treatment. Another tool for assessing the need for anti-osteoporotic therapy is to calculate the 10-year probability of a major fracture using the fracture risk assessment tool (FRAX)

Objectives: To assess the need for anti-osteoporotic therapy in women with rheumatoid arthritis (RA) based on the identification of individuals with fragility fractures and high risk of fracture according to FRAX.

Methods: 295 postmenopausal women with RA were included in the study. The average age was $63 \pm 7$ years, the duration of RA was $11[4 ; 16]$ years, the duration of postmenopausal period was 13 [6; 20] years. $121(41 \%)$ patients took glucocorticoids (cumulative dose 9025 [3650; 20720] mg in prednisolone equivalent). A survey was conducted to identify patients with risk factors and a history of fragility fractures. The 10-year probability of a major osteoporotic fracture was assessed using the FRAX tool. In patients treated with glucocorticoids at a dose $>7.5 \mathrm{mg}$ in prednisolone equivalent the estimates of probabilities of a major osteoporotic fracture were adjusted in accordance with the recommendations [1]. Dual-energy X-ray absorptiometry (DXA) of the proximal femur was performed in patients with a moderate risk (probabilities between the upper and lower assessment age-dependent intervention threshold) and the risk of fracture was recalculated with including femoral neck BMD

Results: $83(28.1 \%)$ patients had a prior fragility fracture: $44(14,9 \%)-1,20$ $(6,8 \%)-2$ and $19(6.4 \%)-3$ or more. Vertebral fractures were the most common, they accounted for $62,1 \%$ of all fractures, distal forearm was the second frequent fractures localization (18.2\%). Only $2(0.7 \%)$ women had hip fracture. The average 10-year probability of a major osteoporotic fracture was $17 \%[11$; 28 ] in RA women. 92 (31.2\%) persons were at high risk, 28 (9.5\%) patients - at low risk, and 175 (59.3\%) - at moderate risk. After recalculation of fracture risk with including femoral neck BMD in people at moderate risk $48(16.3 \%)$ patients became at high risk, $9(3.1 \%)$ - at very high risk, and $118(40.0 \%)$ - at low risk. Thus, 149 (50.5\%) RA patients were at very high or high risk and $146(49,5 \%)$ - at low risk of major osteoporotic fracture according to FRAX, among the last - only 3 persons had a history of fragility fracture after age of 40 years.

Conclusion: Our study demonstrated that a half of postmenopausal women with RA had indications for anti-osteoporotic treatment based on the results of a 10-year probability of major fragility fractures using FRAX tool. REFERENCES:

[1] Kanis JA, Johansson H, Oden A, McCloskey EV. Guidance for the adjustment of FRAX according to the dose of glucocorticoids. Osteoporos Int. 2011;22(3):809-816. doi:10.1007/s00198-010-1524-7.

Disclosure of Interests: None declared

DOI: 10.1136/annrheumdis-2021-eular.1332

\section{POS0165 STUDY OF VERTEBRAL AND FEMUR FRACTURE PREVALENCE AND SCANOGRAPHIC BONE ATTENUATION COEFFICIENT OF THE FIRST LUMBAR VERTEBRA IN AN ACADEMIC HOSPITAL SETTING}

Z. Vaghaiwalla ${ }^{1}$, P. Wasserman ${ }^{2}$, G. Kaeley ${ }^{1} .{ }^{1}$ University of Florida College of Medicine- Jacksonville, Rheumatology, Jacksonville, United States of America; ${ }^{2}$ University of Florida College of Medicine- Jacksonville, Radiology, Jacksonville, United States of America

Background: Patients with osteoporosis are prone to suffer fragility fractures leading to an increased risk of future fractures. Detection of osteoporosis has been led by dual energy-ray absorptiometry (DEXA) as the gold standard. However, in acute fracture incidents, DEXA imaging is typically unavailable, and treatment is often delayed. In recent years scanographic bone attenuation coefficient of the first lumbar vertebra (SBAC-L1) on incidental computed tomography (CT) scans of the lumbar spine has been utilized as an alternative assessment of bone density. In particular, SBAC-L1 $\leq 145 \mathrm{HU}$ correlates with osteoporotic bone density on DEXA imaging. ${ }^{2}$

Objectives: This study aims to assess the SBAC-L1 in HU of incidental CT scans in patients presenting with vertebra or femur fragility fractures in an academic hospital as it relates to osteoporosis.

Methods: We conducted a retrospective chart review of patients aged $\geq 45$ admitted to an academic hospital for active vertebral or femur fracture between 1/1/2017- 1/31/2019, excluding traumatic and pathological fractures. Measurement of SBAC-L1 in HU was obtained on incidental CT scan imaging.

Sex, race, prior and post diagnoses of osteoporosis, prior and post DEXA scan orders, use of calcium, vitamin D, and steroids were abstracted.

Results: There were 297 patients with vertebral or femur fractures from 1/1/20171/31/2019. Of 297 patients, 85 deemed appropriate after excluding pathological, traumatic fractures, and lack of CT scan on the admission for fracture.

Average SBAC-L1 for all patients was $100.4 \pm$ standard deviation (SD) of 46.5 $\mathrm{HU} .82 \%$ of all patients had SBAC-L $1 \leq 145 \mathrm{HU}$. Caucasian patients had a lower average SBAC-L1 [99.4 HU] than African American (AA) patients [122.1 HU]. More Caucasian $[n=15]$ had prior diagnoses of osteoporosis than $A A[n=2]$. Caucasian patients were prescribed Calcium and Vitamin $D$ more often $[n=39]$ than $A A[n=3]$. DEXA scan orders were placed more often in Caucasian patients $[n=4]$ than AA patients [ $n=1]$. Osteoporosis medications were prescribed more often in Caucasian patients $[n=8]$ than $A A$ patients $[n=0]$.

Male average SBAC-L1 was $97.4 \mathrm{HU}$ compared to females at 106.3 HU. Females were prescribed calcium and vitamin $D$ more often $[n=38]$ than males $[n=8]$. DEXA scan orders were placed more frequently in females $[n=5]$ than males $[n=2]$. Osteoporosis medications were prescribed more often in females $[n=9]$ than male patients $[n=0]$.

Table 1. Study Characteristics

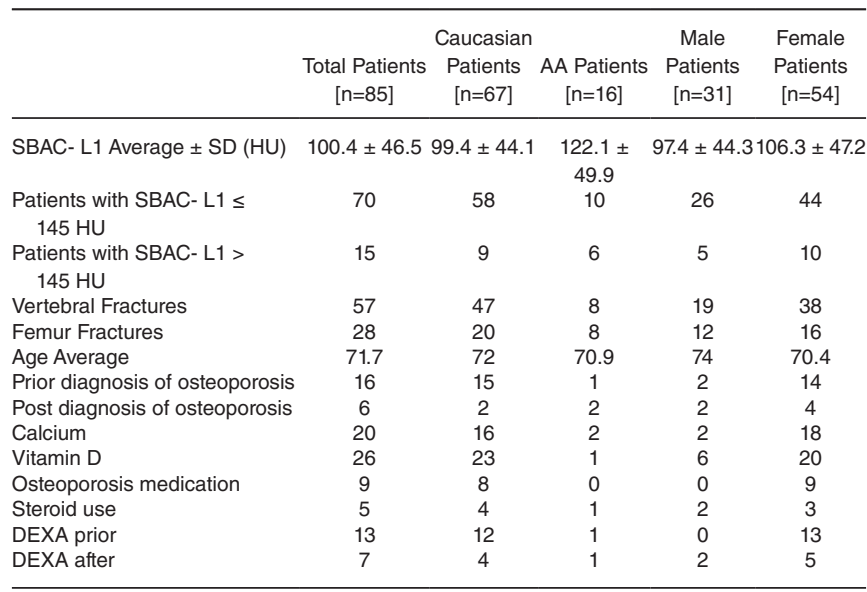

Conclusion: 70 of 85 patients with incidental fragility fractures exhibited SBAC-L1 $\leq 145 \mathrm{HU}$. This is consistent with osteoporotic bone density on DEXA imaging supporting the use of SBAC-L1 measurement to corroborate the diagnosis osteoporosis. SBAC-L $1 \leq 145 \mathrm{HU}$ can be utilized to increase inpatient diagnostic confidence of osteoporosis in the setting of acute fragility fractures to allow initiation of treatment prior to awaiting outpatient DEXA imaging.

The results here also highlight health disparities present among African Americans and males of all races when considering bone health.

\section{REFERENCES:}

[1] Lewiecki, E. Michael, et al. "Healthcare policy changes in osteoporosis can improve outcomes and reduce costs in the United States." JBMR plus 3.9 (2019): e10192.

[2] Fauny, Marine, et al. "Study of vertebral fracture and Scanographic Bone Attenuation Coefficient in rheumatoid arthritis and ankylosing spondylitis vs. controls." Scientific reports 9.1 (2019): 1-9.

Disclosure of Interests: None declared

DOI: 10.1136/annrheumdis-2021-eular.1475

\section{POS0166 ASSOCIATION OF TRABECULAR BONE SCORE AND FAT DISTRIBUTION IN MOROCCAN RHEUMATOID} ARTHRITIS PATIENTS

H. Azzouzi ${ }^{1}$, O. Lamkhanat ${ }^{1}$, I. Linda ${ }^{1} .{ }^{1}$ Mohammed VI University Hospital, Mohammed I University, Faculty of Medicine, Rheumatology, Oujda, Morocco

Background: The association between body composition and bone microarchitecture reflected by trabecular bone score (TBS) has been studied lately (1) In rheumatoid arthritis (RA), this association, especially with fat mass, needs further confirmation.

Objectives: The purpose of the present work was to study the association of TBS with fat distribution in RA.

Methods: We designed a cross-sectional study of patients with RA diagnosis according to ACR-EULAR 2010 classification recruited from first January 2021. Those with prior cancer, hyperparathyroidism, hyperthyroidism, diabetes chronic kidney disease, cirrhosis, and a body mass index (BMI) of more than $36 \mathrm{~kg} / \mathrm{m}^{2}$ were excluded. TBS, total body fat mass (BFM), and its distribution (gynoid (GFM), android (AFM), visceral (VFM), limbs (LFM), trunk (TFM)) were measured with Dual-energy X-ray absorptiometry (Hologic). Clinical data and laboratory tests of the same day of the DXA scan were analyzed. The associations between TBS, BFM, and its distribution were studied by correlations and multiple linear regressions using SPSS 20.

Results: Sixty-nine patients were included. Their mean age was $49.86 \pm 14.33$ years, mean DAS28CRP was $2.56 \pm 1.27$, and mean disease duration was 14.84 \pm 10.99 years. Sixty-two $(89.9 \%)$ were women, with $30(48.4 \%)$ had menopause. The mean BMI was $26.46 \pm 5.26 \mathrm{~kg} / \mathrm{m}^{2}$, and 41 patients were obese $(59.4 \%)$ 